\title{
Hétérogénéité sociale de déclaration de l'état de santé et mesure des inégalités de santé
}

\author{
Marion Devaux, Florence Jusot, \\ Catherine Sermet et Sandy Tubeuf*
}

\section{- Introduction}

En France, comme dans de nombreux pays, le constat de l'existence de fortes inégalités sociales de santé a aujourd'hui été largement dressé (Leclerc et al., 2000; Cambois et Jusot, 2007). Même si une grande partie des recherches s'oriente à présent vers l'analyse des causes de ces inégalités, leur mesure reste un enjeu important de santé publique, notamment pour l'évaluation des politiques mises en œuvre pour les réduire (Aïach, 2000). Ainsi, la loi du 9 août 2004 relative à la politique de santé publique a fixé comme objectif préalable « d'identifier les meilleurs instruments de mesure des inégalités et des discriminations liées à l'origine » afin de poursuivre son trentequatrième objectif, visant «à réduire les inégalités devant la maladie et la mort par une augmentation de l'espérance de vie des groupes confrontés aux situations précaires ».

Or, il reste encore aujourd'hui un large champ de réflexion à mener sur les outils de mesure des inégalités sociales de santé. La méthode utilisée pour leur mesure semble en effet avoir une influence sur l'ampleur des inégalités sociales de santé. Par exemple, la France est le pays d'Europe ayant les plus fortes inégalités sociales de santé lorsque celles-ci sont mesurées par le risque relatif de mortalité prématurée des manuels par rapport aux non manuels (Kunst et al., 2000), alors qu'elle occupe une position moyenne lorsque les inégalités de santé en fonction du revenu sont mesurées à l'aide d'un indice de concentration fondé sur un indicateur de santé perçue (Van Doorslaer et Koolman, 2004). Ainsi, la mesure de l'état de santé, la mesure de la situation sociale et le choix des indicateurs d'inégalités constituent des enjeux importants dans l'analyse des inégalités sociales de santé (Girard, Cohidon et Briançon, 2000; Leclerc et Chastang, 2000; Couffinhal, Dourgnon et Tubeuf, 2004). Dans ce cadre, cet article propose de s'intéresser au rôle de l'instrument choisi pour mesurer l'état de santé, sur l'ampleur des inégalités sociales de santé.

\footnotetext{
* Marion Devaux : statisticienne économiste et Catherine Sermet, directrice adjointe, Irdes. Florence Jusot : maître de conférences en sciences économiques à l'université Paris Dauphine, chercheuse au Legos-Leda et à l'Irdes.

Sandy Tubeuf : doctorante en économie au Greqam Aix-Marseille.
} 
L'état de santé peut être approché par de nombreux indicateurs (mortalité, morbidité, limitations fonctionnelles...). Les indicateurs d'état de santé se distinguent tout d'abord des indicateurs de mortalité puisqu'ils mesurent autant la qualité de vie que le statut vital. Si l'on se limite aux indicateurs de santé, ces derniers peuvent être classés en trois catégories (Blaxter, 1985 ; Sermet et Cambois, 2002). Le modèle subjectif regroupe les indicateurs d'état de santé perçu, les symptômes et les échelles de qualité de vie. Selon le modèle médical ou biologique, l'état de santé peut être évalué par les maladies diagnostiquées ou déclarées et par des anomalies de l'examen clinique, physiologique ou psychiatrique. Dans ce cas, un mauvais état de santé correspond à la non-conformité à une norme physiologique ou psychique. Enfin, selon le modèle fonctionnel et social, l'état de santé est évalué par des indicateurs de limitations fonctionnelles ou de restrictions d'activité. Un mauvais état de santé est alors défini comme l'inaptitude à assurer un rôle social et à réaliser des tâches normales. Ainsi, ces indicateurs permettent l'approche de différentes dimensions de l'état de santé, médicale, fonctionnelle ou de qualité de vie. Enfin, outre la dimension elle-même, la nature déclarative ou diagnostiquée de l'indicateur conduit également à des différences de mesure de l'état de santé.

Or l'ensemble des indicateurs ne décrit pas de la même manière les inégalités de santé. Par exemple, les inégalités d'état de santé entre catégories sociales et groupes de revenus, que celles-ci soient mesurées par des indices de concentration ou par les odds ratios associés aux caractéristiques sociales, sont plus importantes lorsque la santé est mesurée par un indicateur de santé perçue que lorsque celle-ci est mesurée par la présence de maladie chronique ou les limitations fonctionnelles (Devaux et al., 2007). Deux explications à ces différences peuvent être proposées. Ces indicateurs ne faisant pas référence aux mêmes dimensions de la santé, ils doivent nécessairement conduire à une mesure différente des inégalités si les différences sociales de santé sont effectivement d'ampleur différente selon la dimension de la santé considérée ${ }^{1}$. Par ailleurs, on peut penser que chaque indicateur est sujet à une hétérogénéité sociale de déclaration, c'est-à-dire à des différences de déclaration selon les groupes sociaux à «état de santé comparable», différences qui sont également qualifiées de biais de déclaration dans la littérature.

Un courant de recherche s'est récemment intéressé aux biais de déclaration liés à la santé subjective, qui est la mesure d'état de santé la plus régulièrement recueillie dans les enquêtes menées auprès des ménages ${ }^{2}$. Même si cet indicateur est un bon prédicteur de la mortalité (Idler et Benyamini, 1997) et de la consommation de soins (DeSalvo et al., 2005), il semble qu'il soit

$\overline{1 .}$ On peut par exemple supposer que certains groupes professionnels sont exposés à des risques spécifiques de limitations fonctionnelles sans pour autant avoir un risque supérieur de souffrir d'une maladie chronique.

2. Elle consiste généralement en une échelle catégorielle ordinale en cinq points selon laquelle les individus jugent leur état de santé en plusieurs modalités (très mauvais, mauvais, moyen, bon, très bon). 
le résultat d'un processus complexe d'agrégation et de synthèse de plusieurs informations qu'un individu a sur sa santé. En premier lieu, la santé perçue intègre la morbidité connue par l'individu, qui dépend non seulement des maladies et des limitations fonctionnelles dont il souffre, mais aussi des problèmes de santé qui ont été diagnostiqués et pour lesquels il est traité, et donc par là, de ses interactions avec les professionnels de santé. Cette mesure étant subjective, elle intègre ensuite la norme de bonne santé de l'individu et ses attentes en termes de santé, qui sont liées à ses connaissances sur la santé et à son environnement social et culturel. Ainsi, plusieurs travaux ont mis en évidence, des régularités de discordance entre la perception de l'état de santé et des indicateurs de santé jugés plus objectifs. La littérature recense quatre grands ensembles de facteurs affectant le jugement que les individus ont de leur état de santé et donc la déclaration de leur état de santé perçu. Un premier ensemble de biais de déclaration est lié à la nature des pathologies dont souffre l'individu. Par exemple, Van Doorslaer et Gerdtham (2002) observent que les hommes souffrant d'hypertension déclarent un meilleur état de santé que les femmes, à risque de décès équivalent. L'âge et le genre sont également source d'hétérogénéité de déclaration. Par exemple, les femmes rapporteraient une plus mauvaise santé que les hommes pour les mêmes niveaux d'incapacités, ce que Moesgaard et al. (2000) expliquent par le fait qu'elles auraient des normes de bonne santé plus exigeantes. Par ailleurs, Baron-Epel et Kaplan (2001) montrent que les personnes âgées jugent leur santé plus favorablement que les plus jeunes. Des biais de déclaration liés au statut socio-économique ont ensuite été mis en évidence. En France, la déclaration de la santé perçue semble affectée par des biais d'optimisme chez les riches et les plus pauvres, à état de santé clinique donné (Etilé et Milcent, 2006). Enfin, la perception de la santé semble dépendre de caractéristiques culturelles. Par exemple, une étude australienne a montré que la population indigène déclare être en meilleure santé que la population générale malgré des taux d'incidence de problèmes de santé graves bien plus élevés (Mathers et Douglas, 1998).

Les indicateurs de santé déclarée, autres que la santé perçue, semblent également souffrir de biais de déclaration, culturels et sociaux. Un exemple classique est celui de la région du Kerala en Inde, qui a le plus haut taux d'alphabétisation, où la morbidité déclarée est plus importante que dans le reste du pays, alors même que le taux de mortalité y est le plus faible (Murray et Chen, 1992). Plusieurs travaux ont ainsi mis en évidence une sous-déclaration des maladies dans les groupes de faibles niveaux d'éducation, de faible niveau de revenu ou dans les catégories sociales les plus basses (Mackenbach et al., 1996; Elstad, 1996; Murray et Chen, 1992). De même, à partir de données israéliennes, Shmueli $(2002$; 2003) montre une hétérogénéité de déclaration de l'état de santé liée à l'âge, au sexe, à l'éducation, à l'origine ethnique et à la pratique religieuse, variable suivant les indicateurs de santé utilisés : une échelle visuelle analogique (HR-QOL), une échelle de qualité de vie (SF-36), la santé perçue (Shmueli, 2002) et le nombre de maladies chroniques (Shmueli, 2003). 
L'ensemble de ces biais de déclaration liés aux caractéristiques socio-économiques, démographiques, pathologiques ou culturelles sont aujourd'hui reconnus comme un obstacle important pour la comparaison interindividuelle des niveaux de santé déclarée (Bound, 1990) et pour l'analyse des inégalités sociales de santé (Elstad, 1996; Mackenbach et al., 1996; Jusot, Rochaix et Tubeuf, 2005 ; Etilé et Milcent, 2006).

En France, peu de travaux se sont intéressés à cette question. Seuls les biais de déclaration de la santé perçue liés au revenu ont été étudiés (Etilé et Milcent, 2006). Les biais de déclaration affectant les autres indicateurs de santé restent donc à étudier dans le contexte français, puisque différents travaux soulignent l'importance des contextes nationaux (Etilé et Milcent, 2006; Dourgnon et Lardjane, 2007).

Pour étudier les biais de déclaration, l'approche la plus courante consiste à supposer que certains indicateurs sont plus objectifs et permettent mieux d'approcher le «vrai état de santé». Les biais de déclaration correspondent alors à l'écart entre l'état de santé mesuré par les indicateurs jugés «subjectifs » et les indicateurs jugés «objectifs » (Elstad, 1996; Mackenbach et al., 1996; Van Doorslaer et Gerdtham, 2002; Etilé et Milcent, 2006; Perronnin, Tubeuf et Rochaix, 2006). Comme elle privilégie un ou quelques indicateurs jugés plus objectifs, cette approche ne rend pas compte du caractère multidimensionnel du concept de santé, qui ne peut être appréhendé qu'en considérant l'ensemble des indicateurs de santé. Une approche alternative, proposée par Shmueli $(2002 ; 2003)$, consiste à construire un score de santé à partir de plusieurs indicateurs, sans juger de leur objectivité relative, puis à analyser les biais de déclaration à partir de la discordance entre ce score et chacun des indicateurs de santé qu'il utilise.

Suivant cette seconde approche, ce travail propose d'analyser l'hétérogénéité de déclaration liée à la situation économique et sociale affectant plusieurs indicateurs de santé à partir de l'Enquête Santé 2002-2003 de l'Insee.

\section{Données}

\section{L'enquête Santé 2002-2003}

Les données utilisées sont issues de l'Enquête Santé 2002-2003. Cette enquête, qui s'inscrit dans un ensemble d'enquêtes menées régulièrement tous les dix ans depuis 1960, a été réalisée par l'Insee. Elle porte sur un échantillon représentatif de ménages tirés au sort à partir des données du recensement. L'ensemble des personnes composant le ménage est interrogé ; l'échantillon comprend environ 40000 personnes. 


\section{La mesure de l'état de santé}

L'Enquête Santé 2002-2003 comprend de nombreuses questions sur l'état de santé des personnes enquêtées. Pour cette étude, nous avons utilisé les trois questions du mini-module préconisé par Eurostat ${ }^{1}$ et la Direction générale de la santé et de la protection des consommateurs ${ }^{2}$ (DG SANCO) portant sur la santé perçue, les maladies chroniques et les limitations fonctionnelles ainsi que, le score de santé mentale du SF-36. Chacun de ces indicateurs permet d'apprécier une des dimensions de la santé proposée par Blaxter (1985) dans sa typologie. L'indicateur de santé perçue du mini-module européen, de même que le score de santé mentale du SF-36 mesurent l'état de santé subjectif, l'indicateur du mini-module européen portant sur les problèmes de santé chroniques apprécie l'état de santé des personnes enquêtées selon le modèle médical. Enfin, la santé fonctionnelle est mesurée au travers de l'indicateur de limitation d'activité du mini-module.

L'indicateur d'état de santé perçu du mini-module européen correspond à la question : «Comment est votre état de santé général ?»; les réponses possibles sont : «très bon», «bon», «moyen», «mauvais », «très mauvais ». Cet indicateur est dichotomisé en opposant les individus ayant déclaré un état de santé général moyen, mauvais ou très mauvais à ceux qui rapportent un état de santé bon ou très bon. 22,3\% des individus de l'échantillon déclarent avoir un mauvais état de santé perçu.

L'indicateur de santé mentale utilisé est construit à partir du score de la dimension «Mental Health» du SF-36 noté MH. En moyenne les individus ont un score de 66,7 sur 100. Ce score est dichotomisé au premier quartile : le quart des individus ayant un score inférieur à 56 qui est la valeur du premier quartile, est en mauvaise santé.

L'existence de maladies chroniques est appréciée à partir de la question générale sur les maladies chroniques du mini-module européen : "Avezvous actuellement une ou plusieurs maladies chroniques?».39,8\% des individus de notre échantillon ont répondu avoir ou souffrir d'un ou plusieurs problèmes de santé chroniques.

Enfin la mesure de la santé fonctionnelle est réalisée avec l'indicateur de limitations d'activité du mini-module européen : «Êtes-vous limité depuis au moins six mois à cause d'un problème de santé dans les activités que les gens font habituellement?» $11,4 \%$ des individus déclarent avoir une limitation d'activité.

1. Voir dans ce numéro «L'Enquête européenne par interview sur la santé (EHIS) » de Didier Dupré et Albane Gourdol.

2. http://ec.europa.eu/health/ph_information/dissemination/reporting/diagramme_ehss_en.pdf, consulté le 24 octobre 2007. 


\section{La mesure de la situation économique et sociale}

Parmi les nombreuses informations socio-économiques individuelles, disponibles dans cette enquête, les caractéristiques suivantes ont été retenues : l'âge, le sexe, la composition du ménage, le niveau d'éducation, le revenu par unité de consommation, la profession et catégorie sociale, et enfin, le statut d'activité.

L'âge est regroupé en six classes : 18-24 ans ; 25-39 ans ; 40-49 ans ; 50-59 ans; 60-74 ans; 75-85 ans.

Le niveau d'éducation déclaré, repéré par le plus haut diplôme obtenu, permet de construire quatre catégories : les personnes n'ayant aucun diplôme, les personnes ayant un diplôme inférieur au baccalauréat général ou technique, les personnes ayant un diplôme équivalent au baccalauréat général ou technique, et les personnes ayant un diplôme d'études supérieures.

Le revenu par unité de consommation du ménage correspond au revenu total déclaré par le ménage (issu d'une déclaration exacte ou d'une imputation dans le cas d'une déclaration en tranches), divisé par le nombre d'unités de consommation du ménage. L'échelle d'équivalence utilisée afin de prendre en compte les rendements d'échelle au sein du ménage, est l'échelle OCDE qui attribue un poids de 1 à la première personne du ménage, un poids de 0,5 aux autres adultes et un poids de 0,3 aux enfants âgés de moins de 14 ans. Le revenu par unité de consommation est modélisé en quatre classes correspondant aux quatre quartiles de revenu par unité de consommation.

Afin de caractériser le statut social des individus, nous avons retenu la profession et catégorie sociale individuelle à un chiffre, codée à partir de la profession actuelle ou la dernière profession des enquêtés. Cette variable permet de définir six classes sociales correspondant aux agriculteurs, indépendants, cadres, professions intermédiaires, employés et ouvriers, une catégorie supplémentaire correspondant aux personnes n'ayant jamais travaillé.

\section{Échantillon étudié}

Cette analyse est menée sur un échantillon de 20145 individus, restreint aux adultes âgés de 18 à 85 ans ayant répondu à l'ensemble des questions sur la santé. Cet échantillon est donc composé de personnes ayant accepté de participer aux trois visites de l'enquête et ayant renvoyé leur autoquestionnaire sur l'état de santé. L'exclusion des personnes âgées de moins de 18 ans s'explique par la volonté d'étudier des personnes qui, d'une part, ont une situation sociale pouvant être qualifiée et, d'autre part, qui ont répondu elles-mêmes aux questions sur leur état de santé. L'exclusion des personnes âgées de plus de 85 ans est justifiée par les spécificités de l'état de santé des populations très âgées, ainsi que par la moindre fiabilité des données déclaratives pour ces populations. Le tableau 1 présente les caractéristiques de l'échantillon étudié. 
Tableau 1 : Description de l'échantillon

\begin{tabular}{|c|c|c|}
\hline Variables & Effectif & Pourcentage \\
\hline \multicolumn{3}{|l|}{ Sexe } \\
\hline Femme & 10662 & $52,9 \%$ \\
\hline Homme & 9483 & $47,1 \%$ \\
\hline \multicolumn{3}{|l|}{ Classe d'âge } \\
\hline $18-24$ ans & 2326 & $11,5 \%$ \\
\hline $25-39$ ans & 5879 & $29,2 \%$ \\
\hline $40-49$ ans & 4261 & $21,2 \%$ \\
\hline $50-59$ ans & 3586 & $17,8 \%$ \\
\hline $60-74$ ans & 3153 & $15,7 \%$ \\
\hline $75-85$ ans & 940 & $4,7 \%$ \\
\hline \multicolumn{3}{|l|}{ Type de ménage } \\
\hline Personne seule & 2725 & $13,5 \%$ \\
\hline Couple & 6144 & $30,5 \%$ \\
\hline Couple avec enfant & 9407 & $46,7 \%$ \\
\hline Famille monoparentale & 1097 & $5,4 \%$ \\
\hline Famille autre & 772 & $3,8 \%$ \\
\hline \multicolumn{3}{|l|}{ Niveau d'études } \\
\hline Sans diplôme & 2709 & $13,4 \%$ \\
\hline Diplôme inférieur au baccalauréat & 8677 & $43,1 \%$ \\
\hline Baccalauréat & 3445 & $17,1 \%$ \\
\hline Diplôme supérieur au baccalauréat & 5314 & $26,4 \%$ \\
\hline \multicolumn{3}{|l|}{ Revenu } \\
\hline $1^{\text {er }}$ quartile du revenu & 4224 & $21,0 \%$ \\
\hline $2^{\mathrm{e}}$ quartile du revenu & 4983 & $24,7 \%$ \\
\hline $3^{\mathrm{e}}$ quartile du revenu & 5286 & $26,2 \%$ \\
\hline $4^{\mathrm{e}}$ quartile du revenu & 5652 & $28,1 \%$ \\
\hline \multicolumn{3}{|l|}{ Profession } \\
\hline Agriculteur & 667 & $3,3 \%$ \\
\hline Indépendant & 1047 & $5,2 \%$ \\
\hline Cadre & 2853 & $14,2 \%$ \\
\hline Intermédiaire & 4410 & $21,9 \%$ \\
\hline Employé & 5355 & $26,6 \%$ \\
\hline Ouvrier & 4207 & $20,9 \%$ \\
\hline CSP non renseignée & 1606 & $8,0 \%$ \\
\hline \multicolumn{3}{|l|}{ Activité } \\
\hline Actif occupé & 11898 & $59,1 \%$ \\
\hline Chômeur & 1246 & $6,2 \%$ \\
\hline Étudiant & 1253 & $6,2 \%$ \\
\hline Retraité & 3879 & $19,3 \%$ \\
\hline Personne au foyer & 1417 & $7,0 \%$ \\
\hline Inactif & 452 & $2,2 \%$ \\
\hline \multicolumn{3}{|l|}{ État de santé } \\
\hline Avoir un mauvais état de santé perçu & 4486 & $22,30 \%$ \\
\hline Avoir une maladie chronique & 8022 & $39,80 \%$ \\
\hline Avoir une limitation d'activité & 2292 & $11,40 \%$ \\
\hline $\begin{array}{l}\text { Avoir un mauvais état de santé mental } \\
\text { (score SF-36 «Mental Health») }\end{array}$ & 5143 & $25 \%^{1}$ \\
\hline
\end{tabular}

1. Par définition égal à $25 \%$ puisqu'il correspond au quartile. 


\section{Méthode}

Cette analyse repose sur l'utilisation d'un modèle d'équations structurelles à variable latente de type MIMIC (Multiple Indicators Multiple Index Causes), adapté à l'étude des concepts multidimensionnels tel que la santé (Jones, 2002).

Suivant la méthodologie proposée par Shmueli (2002; 2003), nous supposons l'existence d'une variable latente d'état de santé, non directement mesurable et expliquant la réponse à quatre indicateurs de santé : la santé perçue, les limitations d'activité, les maladies chroniques et la santé mentale. L'utilisation d'un modèle MIMIC permet alors de voir dans quelle mesure ces indicateurs de santé sont le reflet d'une et d'une seule variable latente construite à partir des réponses données à chaque indicateur, puis d'analyser l'hétérogénéité de déclaration en séparant l'effet des variables sociodémographiques sur la santé latente, de leur effet direct sur chacun des indicateurs de santé. Cette méthode permet en effet de mettre en évidence des différences sociales de déclaration en supposant que la contribution des caractéristiques sociodémographiques à l'explication de la santé latente relève des déterminants de l'état de santé, alors que, à santé latente donnée, les effets directs de ces caractéristiques sur les indicateurs de santé peuvent être interprétés comme des différences de déclaration.

\section{La construction d'un indicateur synthétique de santé}

La construction de ce modèle demande donc dans un premier temps, une analyse factorielle des données afin de construire un score de santé continu à partir des quatre indicateurs de santé retenu représentant les différentes dimensions de la santé : l'indicateur d'état de santé perçu, de maladies chroniques et de limitations fonctionnelles du mini-module européen et l'indicateur de santé mentale mesurée dans le SF-36. Cette analyse permet de déterminer de façon empirique le nombre de facteurs pertinents résumant l'information de ces indicateurs, c'est-à-dire le nombre de variables latentes d'état de santé sous-jacent, à partir du critère minimum de la valeur propre, c'est-à-dire que le facteur doit avoir une valeur propre égale au moins à 1 pour être retenu.

\section{L'analyse de l'bétérogénéité de déclaration}

Dans le cas où un seul facteur est retenu, il s'agit ensuite d'estimer un modèle à équations simultanées, la première estimant l'effet des caractéristiques socio-économiques sur l'état de santé latent, résumé par le score de santé, et les autres expliquant les réponses aux indicateurs de santé en fonction de l'état de santé latent. Le score de santé est donc employé à la fois comme variable explicative de la réponse aux indicateurs de santé et comme variable dépendante à expliquer par des déterminants de la santé. 
Tester l'existence de l'hétérogénéité sociale de déclaration de la santé revient alors à tester l'existence d'un effet des variables socio-économiques sur la réponse aux indicateurs, indépendamment de leur effet sur la variable latente de santé. Nous appellerons par la suite l'effet direct sur les indicateurs de santé «biais de déclaration».

Plus formellement, le modèle MIMIC à un seul facteur latent peut être formalisé de la façon suivante :

$$
\begin{aligned}
& \eta=\Gamma^{\prime} Z+\zeta(1) \\
& Y=\Lambda \eta+\beta^{\prime} Z+\varepsilon
\end{aligned}
$$

Le score synthétique de santé est une variable continue notée $\eta$. Le vecteur $\mathrm{Y}^{\prime}=(\mathrm{Y} 1, \mathrm{Y} 2, \mathrm{Y} 3, \mathrm{Y} 4)$ est composé de quatre indicateurs dichotomiques ${ }^{1} \mathrm{de}$ santé. Les caractéristiques socio-économiques sont notées $\mathrm{Z}=(\mathrm{Z1}, \mathrm{Z} 2, \ldots)$. Les termes d'erreurs $\zeta$ et $\varepsilon_{\text {sont supposés non corrélés entre eux mais les }}$ erreurs de mesures $\varepsilon_{=}\left(\varepsilon_{1}, \varepsilon_{2}, \ldots\right)$ sont telles que $\varepsilon_{\mathrm{i}}$ et $\varepsilon_{\mathrm{j}}(\mathrm{i}, \mathrm{j}=1,2, \ldots$ et $\mathrm{i} \neq \mathrm{j})$ peuvent être corrélées.

L'équation (1) correspond à la régression linéaire de la variable latente de santé sur les déterminants socio-économiques. L'équation (2) correspond à l'estimation de l'effet de la variable latente de santé, issue de l'analyse factorielle, sur la déclaration des différents indicateurs de santé. Le vecteur $\Lambda^{\prime}=\left(\lambda_{1}, \lambda_{2} \ldots\right)$ correspond aux contributions de l'indicateur synthétique de santé $\eta$ à la réponse aux indicateurs de santé Yi.

Le vecteur $\Gamma$ correspond aux effets des variables socio-économiques $(\mathrm{Z})$ sur la variable de santé non observable, interprétables comme les déterminants $\mathrm{du}$ « vrai » état de santé, et le vecteur $\beta$ aux effets directs des variables socioéconomiques (Z) sur les indicateurs de santé (Y), interprétables comme des biais sociaux de déclaration. Enfin, la corrélation éventuelle des erreurs de mesure $\varepsilon_{\mathrm{i}}$ et $\varepsilon_{\mathrm{j}}$ permet de prendre en compte des biais de déclaration communs à certains indicateurs et indépendants des caractéristiques sociales.

Cette modélisation permet donc de séparer la contribution des variables sociodémographiques à l'explication de la variable latente de santé, de leur contribution à chacun des indicateurs de santé et peut être représentée schématiquement comme suit :

$\overline{\text { 1. Y}} 1$ est un indicateur de mauvais état de santé perçu, Y2 un indicateur de déclaration de maladies chroniques, Y3 un indicateur de déclaration de limitations d'activités et Y4 un indicateur de déclaration de mauvaise santé mentale. 
Figure 1 : Modèle MIMIC

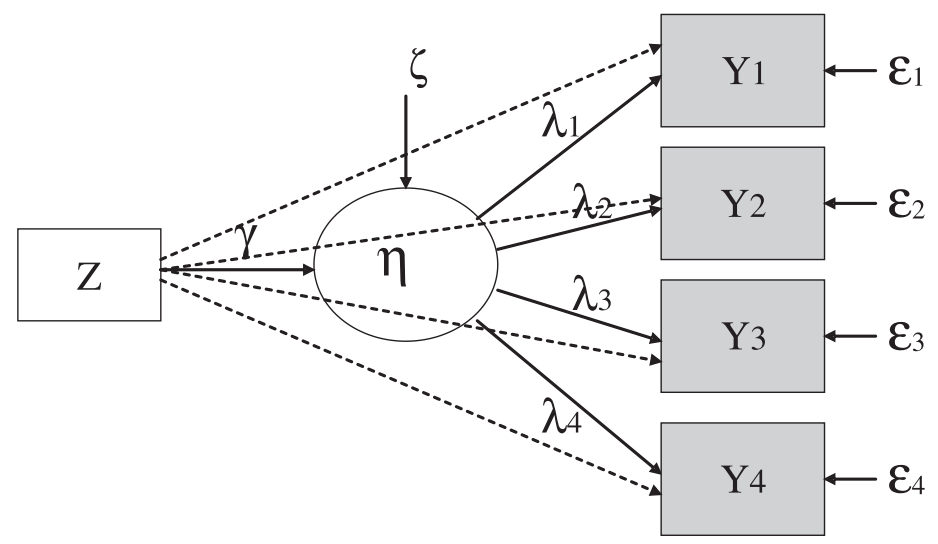

Les équations (1) et (2) sont estimées simultanément. Les paramètres estimés dans l'équation (1) sont des coefficients de régression linéaire, le score de santé étant une variable continue, et les coefficients de l'équation (2) sont des coefficients de modèle probit car les Yi sont des variables catégorielles. Les estimations ont été réalisées sous le logiciel M-Plus. L'ajustement du modèle aux données a été évalué grâce au critère RMSEA (Root Mean Square Error of Approximation), l'ajustement ayant été jugé comme satisfaisant en dessous du seuil de 0,05 .

\section{口 Résultats}

\section{La construction d'un indicateur synthétique unique de mauvaise santé}

L'analyse factorielle exploratoire sur les quatre indicateurs de santé a mis en évidence l'existence d'un seul facteur latent résumant la santé selon le critère minimal de la valeur propre. Celui-ci représente $62 \%$ de l'inertie totale. L'analyse factorielle confirmatoire a confirmé la bonne adéquation aux données d'un modèle à un facteur latent, l'indicateur RMSEA étant égal à 0,031.

La variable latente mise en évidence correspond à un indicateur synthétique continu mesurant la mauvaise santé. Elle est corrélée positivement aux indicateurs de santé suivants : «déclarer un mauvais état de santé perçu», «déclarer une maladie chronique», «déclarer une limitation d'activité» et «avoir une mauvaise santé mentale».

Le modèle MIMIC a ensuite été estimé conduisant, là encore, à un ajustement satisfaisant, le critère RMSEA étant alors égal à 0,007 .

Deux séries de résultats ont été mises en évidence, l'une relative aux déterminants de l'état de santé latent, l'autre aux biais de déclaration affectant les différents indicateurs de santé. 


\section{Les déterminants de l'état de santé latent}

Les résultats présentés dans la colonne «santé latente» du tableau 2 correspondent aux estimations de la régression linéaire de la variable latente de mauvaise santé sur les caractéristiques socio-économiques individuelles. Le sexe, l'âge, le type de ménage, le niveau d'études, le revenu et le niveau social ont tous une influence significative sur la santé latente. Ainsi, la mauvaise santé augmente avec l'âge et les hommes sont en meilleure santé que les femmes. Les personnes seules sont en moins bonne santé que celles vivant en couple sans enfant, elles-mêmes en moins bonne santé que les personnes vivant en couple avec enfants. La mauvaise santé diminue lorsque le niveau d'études augmente. De même, elle diminue avec le niveau de revenu et le niveau social mesuré par la profession et la catégorie sociale. Enfin, les chômeurs, les retraités, les inactifs et les personnes au foyer sont en moins bonne santé que les actifs occupés.

\section{Les biais de déclaration}

La seconde série de résultats concerne les déterminants de la réponse donnée aux quatre indicateurs de santé (colonnes 3 à 7 du tableau 2).

Les résultats présentés dans la ligne «santé latente» montrent tout d'abord que l'état de santé latent contribue significativement à la déclaration de l'indicateur de maladies chroniques (coefficient $=0,609$ ), de limitations d'activité (coefficient $=0,756)$ et de santé mentale (coefficient $=0,54)$, le coefficient associé à la santé latente dans l'estimation de la santé perçue étant posé à 1 par construction.

Les résultats révèlent ensuite l'existence de différents biais de déclaration affectant ces indicateurs. D'une part, la corrélation négative et significative des erreurs de mesure attachées à l'indicateur de santé mentale et la déclaration de maladie chronique suggère un biais de déclaration spécifique attaché à ces deux indicateurs indépendamment des caractéristiques sociodémographiques. D'autre part, les effets directs de certaines caractéristiques sur les indicateurs de santé, à santé latente donnée, suggèrent l'existence de biais de déclaration liés à la fois aux caractéristiques démographiques des répondants et à leur situation économique et sociale.

Il apparaît ainsi, qu'à état de santé latent donné, les femmes déclarent davantage de maladies chroniques et davantage de problèmes de santé mentale que les hommes. Quant aux personnes âgées, elles déclarent davantage de problèmes de santé chroniques et déclarent une meilleure santé mentale. Les personnes vivant seules ou en familles monoparentales par rapport aux personnes en couple, déclarent davantage de problèmes de santé mentale; elles déclarent aussi un état de santé perçu plus mauvais. À l'inverse, elles déclarent avoir moins de maladies chroniques que les personnes vivant en couple. 
Tableau 2 : Déterminants de la mauvaise santé latente et de la probabilité de déclarer un mauvais état de santé selon chacun des indicateurs

\begin{tabular}{|c|c|c|c|c|c|c|c|c|c|}
\hline \multirow[t]{2}{*}{$\begin{array}{l}\text { Caractéristiques } \\
\text { de l'individu }\end{array}$} & \multicolumn{2}{|c|}{$\begin{array}{c}\text { Mauvaise } \\
\text { santé latente }\end{array}$} & \multicolumn{2}{|c|}{$\begin{array}{l}\text { Mauvais état } \\
\text { de santé perçu }\end{array}$} & \multicolumn{2}{|c|}{$\begin{array}{l}\text { Avoir une } \\
\text { maladie } \\
\text { chronique }\end{array}$} & \multicolumn{2}{|c|}{$\begin{array}{c}\text { Avoir des } \\
\text { limitations } \\
\text { d'activité }\end{array}$} & $\begin{array}{c}\text { Mauvaise } \\
\text { santé mentale }\end{array}$ \\
\hline & Estim. & $T$-test & Estim. & T-test & Estim. & T-test & Estim. & T-test & Estim. $T$-test \\
\hline \multicolumn{10}{|l|}{ Sexe } \\
\hline Homme & Ref. & Ref. & & & Ref. & Ref. & & & \multirow{2}{*}{$\begin{array}{rr}\text { Ref. } & \text { Ref. } \\
0,296 & 13,371\end{array}$} \\
\hline Femme & 0,087 & 3,734 & & & 0,05 & 2,293 & & & \\
\hline \multicolumn{10}{|l|}{ Âge } \\
\hline $18-24$ ans & $-0,262$ & $-5,697$ & \multirow{6}{*}{\multicolumn{2}{|c|}{ Ref. Ref. }} & & & \multirow{6}{*}{ Ref. } & \multirow{6}{*}{ Ref. } & \multirow{6}{*}{\begin{tabular}{lr}
\multicolumn{1}{c}{ Ref. } & Ref. \\
$-0,07$ & $-2,429$ \\
$-0,174$ & $-5,289$ \\
$-0,31$ & $-5,426$ \\
$-0,273$ & $-3,979$ \\
\end{tabular}} \\
\hline $25-39$ ans & Ref. & Ref. & & & Ref. & Ref. & & & \\
\hline $40-49$ ans & 0,284 & 9,911 & & & & & & & \\
\hline $50-59$ ans & 0,503 & 15,227 & & & 0,113 & 3,666 & & & \\
\hline $60-74$ ans & 0,53 & 9,51 & & & 0,335 & 6,22 & & & \\
\hline $75-85$ ans & 0,88 & 13,32 & & & 0,301 & 4,427 & & & \\
\hline \multicolumn{10}{|l|}{ Type de ménage } \\
\hline Personne seule & 0,071 & 2,336 & \multirow{5}{*}{\multicolumn{2}{|c|}{$\begin{array}{rr}\text { Ref. } & \text { Ref. } \\
0,149 & 2,401\end{array}$}} & & & \multirow{5}{*}{ Ref. } & \multirow{5}{*}{ Ref. } & $0,204 \quad 6,662$ \\
\hline Couple sans enfant & Ref. & Ref. & & & Ref. & Ref. & & & Ref. Ref. \\
\hline Couple avec enfant & $-0,054$ & $-2,157$ & & & & & & & \\
\hline Famille monoparentale & $-0,088$ & $-1,466$ & & & & & & & $0,243 \quad 4,748$ \\
\hline Famille autre & 0,024 & 0,456 & & & $-0,145$ & $-2,804$ & & & \\
\hline \multicolumn{10}{|l|}{ Niveau d'études } \\
\hline Aucun diplôme & Ref. & Ref. & \multirow{4}{*}{\multicolumn{2}{|c|}{$\begin{array}{rr}\text { Ref. } & \text { Ref. } \\
-0,119 & -3,682 \\
-0,108 & -2,498\end{array}$}} & Ref. & Ref. & Ref. & Ref. & Ref. \\
\hline Diplôme inférieur bac & $-0,1$ & $-2,993$ & & & & & & & \\
\hline Diplôme niveau bac & $-0,237$ & $-5,469$ & & & & & & & \\
\hline Diplôme supérieur au bac & $-0,468$ & $-11,477$ & & & 0,185 & 4,691 & 0,18 & 3,48 & \\
\hline Revenu & & & & & & & & & \\
\hline $1^{\text {er }}$ quartile du revenu & Ref. & Ref. & Ref. & Ref. & Ref. & Ref. & Ref. & Ref. & Ref. \\
\hline $2^{\mathrm{e}}$ quartile du revenu & $-0,074$ & $-2,563$ & & & 0,068 & 2,443 & & & \\
\hline $3^{\mathrm{e}}$ quartile du revenu & $-0,194$ & $-6,452$ & & & 0,124 & 4,226 & & & \\
\hline $4^{\mathrm{e}}$ quartile du revenu & $-0,23$ & $-6,981$ & & & 0,156 & 4,839 & & & \\
\hline Profession & & & & & & & & & \\
\hline Agriculteur & $-0,1$ & $-1,918$ & & & & & & & \\
\hline Indépendant & $-0,149$ & $-3,332$ & & & & & & & \\
\hline Cadre & $-0,298$ & $-6,754$ & & & 0,163 & 3,946 & 0,112 & 2,093 & \\
\hline Intermédiaire & $-0,171$ & $-4,995$ & & & 0,124 & 3,802 & 0,112 & 2,786 & \\
\hline Employé & $-0,077$ & $-2,723$ & & & & & & & \\
\hline Ouvrier & Ref. & Ref. & Ref. & Ref. & Ref. & Ref. & Ref. & Ref. & Ref. \\
\hline CSP non renseignée & $-0,199$ & $-3,046$ & & & & & & & \\
\hline Activité & & & & & & & & & \\
\hline Actif occupé & Ref. & Ref. & Ref. & Ref. & Ref. & Ref. & Ref. & Ref. & Ref. \\
\hline Chômeur & 0,314 & 7,849 & & & & & & & \\
\hline Étudiant & 0,251 & 2,769 & $-0,357$ & $-3,521$ & & & & & \\
\hline Retraité & 0,246 & 5,155 & & & & & 0,198 & 3,605 & \\
\hline Personne au foyer & 0,173 & 4,214 & & & & & & & \\
\hline Inactif & 0,942 & 14,875 & & & & & 0,547 & 8,898 & \\
\hline Seuil/Constante & & & 0,709 & 14,816 & 0,567 & 13,122 & 1,367 & 24,345 & $0,592 \quad 13,252$ \\
\hline Santé latente (CTR norm.) & & & & & 0,609 & 36,355 & 0,756 & 39,859 & $0,54 \quad 33,692$ \\
\hline $\mathrm{R}^{2}$ & 0,246 & & 0,888 & & 0,402 & & 0,568 & & 0,314 \\
\hline Chi deux (WLSMV) & 73,244 & & & & & & & & \\
\hline P-value & 0,0005 & & & & & & & & \\
\hline RMSEA & 0,007 & & & & & & & & \\
\hline Corrélation maladies & -0051 & & & & & & & & \\
\hline chronıques et santé ment & $-0,051$ & & & & & & & & \\
\hline
\end{tabular}

Guide de lecture : la première colonne contient les coefficients de la régression linéaire de la mauvaise santé latente. Les quatre autres colonnes contiennent les coefficients des probit correspondant aux quatre indicateurs de santé modélisant de la mauvaise santé. Ainsi, un signe négatif du coefficient signifie un impact positif sur la bonne santé quel que soit l'indicateur. Le coefficient est significatif si lt-testl > 1,96. Le sexe a un effet significatif sur la variable latente de santé (coefficient $=0,087$ ) : les femmes sont en moins bon état de santé latent que les hommes. A santé latente donnée, les femmes déclarent davantage de maladies chroniques que les hommes (coefficient $=0,05$ ) et elles déclarent une moins bonne santé mentale que les hommes (coefficient $=0,296$ ). 
Le niveau de diplôme et le revenu ont une influence significative sur les variables d'état de santé, à santé latente donnée. Avoir un diplôme inférieur ou équivalent au bac semble significativement lié à un meilleur état de santé perçu. Parallèlement, les individus ayant un diplôme supérieur au bac déclarent davantage de maladies chroniques et de limitations d'activité. Le niveau de revenu a un effet direct et positif sur l'indicateur de maladies chroniques : plus le revenu est élevé, plus les individus déclarent de maladies chroniques. Quant à la profession, les individus cadres ou occupant une profession intermédiaire déclarent davantage de maladies chroniques et de limitations d'activité à santé latente donnée. Enfin, alors que les étudiants déclarent moins de problème de santé générale que les actifs occupés, les retraités et les inactifs déclarent davantage de limitations d'activité.

\section{Discussion}

L'objectif de cette étude était d'analyser les biais sociaux affectant la déclaration de l'état de santé et pouvant donc affecter la mesure des inégalités sociales de santé. Les résultats confirment des différences sociales d'état de santé latent. En outre, nos résultats montrent une hétérogénéité de déclaration à santé latente donnée. Ainsi, à santé latente donnée, les femmes et les personnes âgées déclarent plus souvent des maladies chroniques. Les problèmes de santé mentale sont surdéclarés par les femmes, les personnes isolées et sous-déclarés par les plus âgées. Les inactifs et les retraités déclarent plus fréquemment des limitations d'activité, de même que les cadres. Enfin, les personnes les plus éduquées, aux revenus élevés, les cadres et les professions intermédiaires déclarent plus fréquemment des maladies chroniques tandis que les personnes peu éduquées sous-déclarent la mauvaise santé perçue.

L'approche utilisée, proposée initialement par Shmueli (2002; 2003), a permis de construire un indicateur synthétique de santé latente à partir de quatre indicateurs de santé, puis de séparer l'association entre les caractéristiques sociodémographiques et les indicateurs de santé en une contribution de ces caractéristiques à l'explication de la santé latente et en leur contribution directe à chacun des indicateurs de santé. Cette méthode permet donc de mettre en évidence des biais de déclaration spécifiques dans la mesure où la contribution des caractéristiques sociodémographique à l'explication de la santé latente peut être interprétée comme relevant des déterminants de l'état de santé, alors que les effets directs de ces caractéristiques sur les indicateurs de santé peuvent être interprétés comme des différences sociales de déclaration de l'état de santé. Cette méthodologie et l'interprétation des résultats qui en découlent peuvent toutefois être discutées.

Cette méthode repose sur l'hypothèse de l'existence d'une unique variable latente d'état de santé expliquant la réponse aux différents indicateurs de santé. L'analyse factorielle exploratoire sur les quatre indicateurs de santé 
a montré l'existence d'un seul facteur latent résumant la santé, confirmant ainsi le fait que l'état de santé pouvait être représenté de façon satisfaisante par une seule variable c'est-à-dire une seule dimension. Cependant, ce facteur ne représente que $62 \%$ de l'inertie totale. La variable latente construite par cette méthode ne permet donc pas d'avoir une représentation complète de la santé, qui est un concept largement multidimensionnel.

Cette première hypothèse conduit à interpréter les effets directs des caractéristiques sociodémographiques sur les indicateurs de santé comme des biais de déclaration de l'état de santé. Cependant, ces effets peuvent non seulement correspondre à des biais de déclaration, mais aussi aux effets de certaines caractéristiques sur des dimensions spécifiques de l'état de santé, relevant donc des déterminants de la santé. Par exemple, l'effet spécifique du genre sur le score de santé mentale du SF-36 peut non seulement être dû à une surdéclaration des problèmes de santé mentale par les femmes, mais aussi à une association plus forte entre le genre et cette dimension de l'état de santé comparativement aux autres dimensions, notamment expliquée par la forte différence de prévalence de la dépression entre les femmes et les hommes (Grigoriadis et al., 2007).

Cette méthode identifiant les biais de déclaration à partir des différences d'association entre les caractéristiques sociodémographiques et chacun des indicateurs de santé, elle permet donc d'identifier des biais spécifiques affectant chacun des indicateurs mais ne permet pas d'identifier des biais communs affectant l'ensemble des indicateurs. Ainsi, un biais d'optimisme ou de pessimisme affectant la déclaration des quatre indicateurs retenus et corrélé à une caractéristique sociodémographique particulière ne sera pas identifié comme un biais mais sera confondu avec l'effet de cette caractéristique sur l'indicateur synthétique de santé latente. Toutefois, l'autorisation d'une corrélation des erreurs de mesure attachées à chacun des indicateurs de santé a permis de mettre en évidence un biais de déclaration spécifique attaché à l'indicateur de santé mentale et à l'indicateur de maladie chronique.

La variable latente de santé a été construite à partir de l'information commune aux quatre indicateurs utilisés. L'indicateur synthétique de santé latente est donc par définition dépendant des indicateurs de santé retenus. Afin de tester la stabilité des résultats, nous avons remplacé tour à tour chacun des quatre indicateurs par d'autres indicateurs disponibles dans l'enquête se rapportant à la même dimension de la santé : l'état de santé perçu a été remplacé par le score de santé générale du SF-36, la déclaration des maladies chroniques a été mesurée à partir de la liste de maladies déclarées, la santé fonctionnelle a été appréciée à partir de la déclaration des incapacités et déficiences, enfin la santé mentale a été mesurée à partir du score CES-D. Cette analyse de sensibilité a naturellement conduit à des résultats en partie différents mais la plupart des biais mis en évidence se sont avérés stables. En particulier, cette analyse a confirmé la sous-déclaration de la mauvaise santé perçue par les étudiants et sa surdéclaration par les familles monoparentales; la déclaration plus fréquente des maladies chroniques par 
les personnes âgées, les plus éduquées et les plus riches; la déclaration plus fréquente des problèmes fonctionnels par les retraités, les personnes inactives et les plus éduqués; enfin, la surdéclaration des problèmes de santé mentale par les femmes, les personnes seules ou familles monoparentales et la sous-déclaration par les personnes âgées.

Cette analyse confirme des résultats déjà montrés dans la littérature et suggère l'existence de biais de déclaration jusqu'à présent peu discutés.

Nous mettons en évidence des inégalités sociales de santé mesurées à partir de l'indicateur de santé latente, en accord avec de nombreuses études décrivant une détérioration de l'état de santé avec la catégorie sociale, l'éducation et le revenu (Leclerc et al., 2000; Cambois et Jusot, 2007). Nous montrons ensuite l'existence des biais affectant la déclaration de santé selon les quatre indicateurs considérés : maladie chronique, limitations d'activité, santé perçue et score de santé mentale du SF-36.

Le grand nombre d'effets directs affectant l'indicateur des maladies chroniques suggère que cet indicateur fournit une mesure particulièrement biaisée de la santé selon les caractéristiques sociodémographiques individuelles. En cohérence avec les travaux de Moesgaard et al. (2000), nous montrons que les femmes surdéclarent les maladies chroniques. Cet effet du genre sur la déclaration des maladies peut sans doute être expliqué par un recours aux soins plus fréquent à état de santé donné, une attention plus grande portée aux problèmes de santé, une meilleure connaissance des problèmes de santé expliquée en partie par des problèmes de santé plus fréquents, les femmes ayant un état de santé latent moins bon que les hommes. En cohérence avec les travaux de Shmueli (2003), les résultats suggèrent également une surdéclaration des maladies chroniques par les personnes âgées. Ce phénomène peut, là encore, s'expliquer par une meilleure connaissance des maladies chroniques liée à un recours aux soins plus fréquent. Les personnes les plus éduquées, ayant les plus hauts revenus ainsi que les cadres et professions intermédiaires déclarent plus fréquemment des maladies chroniques à état de santé donné. Ces différences sociales de déclaration, déjà mises en évidence par Mackenbach et al. (1996) et Elstad (1996), peuvent s'expliquer à nouveau par une meilleure information médicale liée à un recours aux soins plus fréquent ou par une plus grande attention portée à la santé par les classes sociales les plus aisées. Par ailleurs, on peut se demander si la notion de maladie chronique est comprise de la même manière par tous les groupes sociaux.

L'indicateur de limitations d'activité fait également apparaître un biais de déclaration lié au niveau d'études et à la profession. Les individus ayant un diplôme supérieur au baccalauréat, les cadres et les professions intermédiaires, bien qu'ils aient une meilleure santé latente que les non diplômés ou que les ouvriers, déclarent plus fréquemment des limitations d'activité. Cette surdéclaration relève peut-être d'une moins grande tolérance aux limitations fonctionnelles et aux restrictions d'activité liées à l'état de santé. De plus, nous observons une surdéclaration des limitations d'activité par les retraités 
et les inactifs. Ce résultat correspond au biais de justification proposé par Bound (1990) selon lequel les personnes justifieraient leur retrait du marché du travail par un mauvais état de santé. Cependant, on peut également penser que les personnes inactives ou préretraitées ont un risque spécifique de souffrir de limitations d'activité, celles-ci expliquant pour beaucoup leur retrait anticipé du marché du travail (Barnay et Debrand, 2006; Blanchet et Debrand, 2007).

Plusieurs biais affectant la déclaration de la santé perçue ont également été mis en évidence. Nous observons que les individus ayant un niveau d'études intermédiaire déclarent moins fréquemment un mauvais état de santé que les personnes les plus éduquées ou sans diplôme. Ce biais d'optimisme par rapport aux individus les plus éduqués peut être le reflet d'une attente en matière de santé croissante avec le niveau d'éducation, comme le suggèrent les travaux de Mackenbach et al. (1996) ou Elstad (1996). Par ailleurs, les étudiants déclarent un meilleur état de santé perçu alors qu'ils ont une moins bonne santé latente que les actifs occupés, en raison notamment d'allergies, de dépression et d'anxiété. Ce biais d'optimisme suggère peut-être qu'ils n'intègrent pas ces problèmes de santé chroniques ou de santé mentale dans l'appréciation de leur état de santé général. Enfin, les individus appartenant à une famille monoparentale déclarent plus fréquemment un mauvais état de santé perçu, à état de santé latent donné. Cette surdéclaration relève peut-être de la plainte ou d'une médicalisation d'un problème social, c'est-à-dire d'une expression d'un mal être social au travers de la déclaration de problème de santé.

Les résultats relatifs au score de santé mentale du SF-36 suggèrent une surdéclaration de ce type de problèmes de santé par les femmes, en cohérence avec les résultats l'analyse menée en Israël par Shmueli (2003). Cependant, ce résultat peut également être dû à un effet spécifique du sexe sur cette dimension de la santé, le risque de dépression ou d'anxiété étant plus fréquent chez les femmes (Grigoriadis et al., 2007). Nous confirmons également le biais de sous-déclaration des problèmes de santé mentale par les personnes âgées montré par Shmueli (2003). Cet effet pourrait être expliqué par des attentes moindres en termes de santé mentale des personnes âgées, en raison notamment des nombreux problèmes de santé liés à l'âge. Cependant, cet effet peut, là encore, non pas être lié à un biais de déclaration mais à un effet de l'âge moins marqué sur la santé mentale que sur les autres dimensions de la santé. Enfin, nous montrons une surdéclaration des problèmes de santé mentale par des personnes seules ou les familles monoparentales, sans doute attribuable en partie à l'influence spécifique de l'isolement sur cette dimension de la santé (Wang, 2004).

Cette analyse souligne donc l'existence d'une hétérogénéité de déclaration liée à la situation sociodémographique affectant l'ensemble des indicateurs considérés. Parmi ces indicateurs, la déclaration de maladies chroniques souffre de nombreux biais, et notamment d'un biais de pessimisme lié à l'éducation, à la profession et aux revenus. En conséquence, cet indicateur ne 
peut pas être considéré comme un bon outil de mesure des inégalités sociales de santé, puisqu'il conduit à sous-estimer leur ampleur. Au contraire, les indicateurs de santé perçue, de limitations d'activité et de santé mentale semblent moins biaisés. Ces indicateurs traduisant différents domaines de la santé, ils peuvent donc être utilisés avantageusement selon les problématiques soulevées. Dans l'objectif d'une surveillance plus globale des inégalités sociales de santé, retenu dans le cadre de la loi du 9 août 2004 relative à la politique de santé publique, l'indicateur d'état de santé perçu apparaît enfin comme un bon outil de mesure. 


\section{Bibliographie}

AÏACH P., (2000), «De la mesure des inégalités : enjeux sociopolitiques et théoriques», in Leclerc A. et al., Les inégalités sociales de santé, La Découverte/Inserm, p. 83-92.

BARNAY T., DEBRAND T., (2006), «L'impact de l'état de santé sur l'emploi des seniors en Europe», Questions d'économie de la santé, Irdes, nº 109.

BARON-EPEL O., KAPLAN G., (2001), «General subjective health status or age-related subjective health status : does it make a difference?», Social Science and Medicine, vol. 53, p. 1373-1381.

BLANCHET D., DEBRAND T., (2007), «Souhaiter prendre sa retraite le plus tôt possible : santé, satisfaction au travail et facteurs monétaires », Économie et Statistique, no 403-404, 2007/12, 39-62.

BLAXTER M., (1985), «A comparison of measures of inequality in morbidity », in John Fox, Health Inequalities in European Countries, Aldershot, Gower.

BOUND J., (1990), «Self-Reported versus Objective Measures of Health in Retirement Models », The Journal of Human resources, 26, p. 107-137.

CAMBOIS E., JUSOT F., (2007), «Ampleur, tendance et causes des inégalités sociales de santé et de mortalité en Europe : une revue des études comparatives », Bulletin Épidémiologique Hebdomadaire, numéro thématique «Les inégalités sociales de santé en France en 2006 : éléments de l'état des lieux», 2007/01/23, 2-3, p. 10-14.

COUFFINHAL A., DOURGNON P., TUBEUF S., (2004), «Outils de mesure des inégalités : quelques débats d'actualité», Santé, Société et Solidarité, p. 163-171.

DESALVO K.B., FAN V.S., MCDONELL M.B., FIHN S.D., (2005), «Predicting Mortality and Healthcare Utilization with a Single Question», Health Services Research, 40, 4, p. 1234-46.

DEVAUX M., DOURGNON P., JUSOT F., LARDJANE S., SERMET C., TUBEUF S., (2007), «Hétérogénéité sociale de mesure de l'état de santé et mesure des inégalités sociales de santé», rapport dans le cadre du programme Drees-MiRe, Inserm, DGS, InVS, INCa, CANAM «Inégalités sociales de santé».

DOURGNON P., LARDJANE S., (2007), «Les comparaisons internationales d'état de santé subjectif sont-elles pertinentes? Une évaluation par les méthodes des vignettesétalons», Économie et Statistique, no ${ }^{\circ}$ 403-404, p. 165-177.

ELSTAD J.I., (1996), «How large are the Differences - Really? Self-reported Long standing Illness Working Class and Middle Class Men», Sociology of Health an Illness, 18,4 , p. 475-498.

ETILÉ F., MILCENT C., (2006), «Income-related reporting heterogeneity in selfassessed health : evidence from France», Health Economics, 15, p. 965-981.

GIRARD F., COHIDON C., BRIANÇON S., (2000), «Les indicateurs globaux de santé», in Leclerc A. et al., Les inégalités sociales de santé, La Découverte/Inserm, p. 163-172.

GRIGORIADIS S., ROBINSON G.E., (2007), «Gender issues in depression», Annals of Clinical Psychiatry, 19 (4), p. 247-255.

IDLER E.L., BENYAMINI Y., (1997), «Self-rated health and mortality : a review of twenty-seven community studies», Journal of Health and Social Behaviour, 38, p. 21-37.

JUSOT F., ROCHAIX L., TUBEUF S., (2005), «Income-Related Health Inequalities in France between 1998 and 2002 : Comparing Trends with Alternative Health Indicators», ECuity III Workshop, Bonn, september. 
KUNST A.E., GROENHOF F., MACKENBACH J.P. et EU WORKING GROUP ON SOCIO-ECONOMIC INEQUALITIES IN HEALTH, (2000), «Inégalités sociales de mortalité prématurée : la France comparée aux autres pays européens», in Leclerc A., Fassin D., Grandjean H., Kaminski M., Lang T., Les inégalités sociales de santé, Paris, La Découverte/Inserm.

LECLERC A., FASSIN D., GRANDJEAN H., KAMINSKI M., LANG T., (2000), Les inégalités sociales de santé, Paris, La Découverte/Inserm.

LECLERC A., CHASTANG J.-F., (2000), «Quantifier les inégalités », in Leclerc A., Fassin D., Grandjean H., Kaminski M., Lang T., Les inégalités sociales de santé, Paris, La Découverte/Inserm, p. 109-122.

MACKENBACH J.P., LOOMAN C.W., VAN DER MEER J.B., (1996), «Differences in the misreporting of chronic conditions, by level of education : the effect on inequalities in prevalence rates », Am. J. Public Health, 86, p. 706-711.

MATHERS C., DOUGLAS R.M., (1998), «Measuring progress in population health and well-being », in Eckersley R. (ed.), Measuring progress : Is life getting better?, Collingwood Vic.

MOESSGAARD IBURG K.M., SALOMON J.A., TANDON A., MURRAY C.J.L., (2002), «Cross-population comparability of physician-assessed and self-reported measures of health », in Murray C.J.L., Salomon J.A., Mathers C., Lopez A.D., WHO, Summary Measures of Population Health : Concepts, Ethics, Measurement and Applications, Geneva, p. 433-448.

MURRAY C.J.L., CHEN L.C., (1992), «Understanding Morbidity Change», Population and Development Review, 18, p. 481-503.

PERRONNIN M., ROCHAIX L., TUBEUF S., (2006), «Construction d'un indicateur continu d'état de santé agrégeant risque vital et incapacité», Questions d'économie de la santé, Irdes, $\mathrm{n}^{\circ} 107$, mai.

RADLOFF L.S., (1977), «The CES-D scale : a self-report depression scale for research in the general population», Applied Psychological measurement, vol. $1 \mathrm{n}^{\mathrm{o}}$ 3, p. 385-401.

SERMET C., CAMBOIS E., (2002), «Mesurer l'état de santé», in Caselli G., Vallin J., Wunsch G., Démographie : analyse et synthèse. III. Les déterminants de la mortalité, Paris, Éditions de l'Ined, 2002/12, vol. III, chapitre 41, p. 25-52.

SHMUELI A., (2002), «Reporting Heterogeneity in the Measurement of Health and Health-Related Quality of Life», Pharmacoeconomics, vol. 20, nº 6, p. 405-412.

SHMUELI A., (2003), «Socio-economic and demographic variation in health and in its measures : the issue of reporting heterogeneity», Social Science and Medicine, 57, p. $125-134$.

VAN DOORSLAER E., KOOLMAN X., (2004), «Explaining the differences in incomerelated health inequalities across European countries», Health Economics, 13, p. 609-628.

VAN DOORSLAER E., GERDTHAM U.G., (2003), «Does inequality in self-assessed health predict inequality in survival by income? - Evidence from Swedish data», Social Science and Medicine, 57, p. 1621-1629.

WANG J.L., (2004), «The difference between single and married mothers in the 12month prevalence of major depressive syndrome, associated factors and mental health service utilization », Social Psychiatry and Psychiatric Epidemiology, 39 (1), p. 26-32. 